\title{
PROJECT-BASED LEARNING AS AN INSTRUCTIONAL TOOL IN TEACHING AD DRAFTING TO STUDENTS MAJORING IN ADVERTISING
}

E. Malyuga1, V. Vetrinskaya1, A. Krouglov2

1RUDN-University (RUSSIAN FEDERATION)

2University College London (UNITED KINGDOM)

As advertising penetrates into all social spheres, it vastly affects the social behaviour of different linguocultural communities. While advertisements have a profound effect on the social medium, it is the extent and the various facets of this influence that grab the headlines of scientific research.

This article aims to consider one of the leading methods of teaching ad drafting to students majoring in Advertising while focusing mainly on the functions and syntactic characteristics of the advertising discourse.

Considering the peculiarities of ad drafting as a class activity, the article brings a focus on the project method as one of the most efficient training frameworks that allows to introduce profession-specific practices into the learning process, promote creativity and foster proactive mindsets in the context of learner-cantered practice-oriented training environment.

The study highlights the theoretical considerations involved in the study of advertisements, specifically centering around their syntax and functions that include attracting attention, informing, persuading, motivating by offering discounts and gifts, and most importantly encouraging the consumer to choose a particular product or service being advertised. The syntactical peculiarities and nomenclature of the basic functions of advertisements are further on analyzed to explain the corresponding teaching and learning implications and clarify the place and role of the project method in ad drafting training.

The authors suggest that while project-based learning implies a lot of creative activity being incorporated into the class, purposeful implementation of projects cannot only be associated with motivational boost, encouragement of personal interest, or a somewhat informal practice of skill transfer: as the article stresses, projects developed as part of the instructional "toolkit" in ad drafting classes should at all times be designed so as to teach students to think strategically. Strategic reasoning, in turn, should be based on the awareness of the functional load behind the language and syntax used in advertising texts. 
Thus, the paper singles out syntactical features inherent in the advertising discourse (such as, for example, brevity, an abundant use of elliptical constructions, or prevalence of imperative sentences) and makes systemized conclusions regarding the project-based methodological routine most suitable for the purposes of ad drafting classes with students majoring in Advertising.

keywords: teaching methods, advertising discourse, project-based learning. 